\title{
Contribution of TLR4 signaling in intermittent hypoxia-mediated atherosclerosis progression
}

\author{
Xianqin Zeng ${ }^{1,2,4}$, Rong Guo ${ }^{1}$, Mei Dong ${ }^{2}$, Julia Zheng ${ }^{3}$, Huili Lin ${ }^{1 *}$ (D) and Huixia Lu $2^{*}$
}

\begin{abstract}
Background: Intermittent hypoxia $(\mathrm{IH})$, a typical character of obstructive sleep apnea (OSA), is related to atherogenesis. However, the role of $\mathrm{IH}$ on atherosclerosis (AS) progression and the mechanisms involved remains poorly understood.

Methods: In the present study, high-fat fed ApoE ${ }^{-/-}$mice were treated with recombinant shRNA-TLR4 lentivirus and exposed to $\mathrm{IH}$. Atherosclerotic lesions on the en face aorta and cross-sections of aortic root were examined by Oil-Red $O$ staining. The content of lipids and collagen of aortic root plaques were detected by Oil-Red $O$ staining and Sirius red staining, respectively. The TLR4, NF-KB p65, a-SMA and MOMA-2 expression in aorta and IL-6 and TNF-a expression in the mice serum were also detected.

Results: Compared with the Sham group, the $\mathrm{IH}$ treated group further increased atherosclerotic plaque loads and plaque vulnerability in the aortic sinus. Along with increased TLR4 expression, enhanced NF-KB activation, inflammatory activity and aggravated dyslipidemia were observed in the IH treated group. TLR4 interference partly inhibited IH-mediated AS progression with decreased inflammation and improved cholesterol levels. Similarly, in endothelial cells, hypoxia/reoxygenation exposure has been shown to promote TLR4 expression and activation of proinflammatory TLR4/NF-kB signaling, while TLR4 interference inhibited these effects.

Conclusions: We found that the $\mathrm{IH}$ accelerated growth and vulnerability of atherosclerotic plaque, which probably acted by triggering the activation of proinflammatory TLR4/NF-KB signaling. These findings may suggest that IH is a risk factor for vulnerable plaque and provide a new insight into the treatment of OSA-induced AS progression.
\end{abstract}

Keywords: Atherosclerosis, Intermittent hypoxia, Toll-like receptor 4, Nuclear factor kappa B, Inflammation

\section{Background}

Atherosclerosis (AS) is a chronic vascular disease. High vulnerability of AS plaque is a common pathologic change of acute coronary syndrome (ACS) and stroke, which account for a large part of deaths [1].

\footnotetext{
*Correspondence: linhuilijinan@aliyun.com; luhuixia@sdu.edu.cn ${ }^{1}$ Department of Cardiology, The Second Affiliated Hospital of Fujian Medical University, Quanzhou 362000, Fujian, People's Republic of China

${ }^{2}$ The Key Laboratory of Cardiovascular Remodeling and Function

Research, Chinese Ministry of Education and Chinese Ministry of Health, The State and Shandong Province Joint Key Laboratory of Translational Cardiovascular Medicine, Department of Cardiology, Qilu Hospital of Shandong University, No. 107, Wen Hua Xi Road, Jinan 250012, Shandong, China

Full list of author information is available at the end of the article
}

Cardiovascular risk factors such as diabetes mellitus, dyslipidemia and hypertension, contribute to AS development. In recent decades, obstructive sleep apnea (OSA) has been recognized as a new risk factor of AS [2, 3]. Patients who suffered from both AS and OSA suffer even worse outcomes [4].

OSA, induced by intermittent partial or complete obstruction of the upper airway during sleep, is a public health problem as it affects one in five adults in western countries [5]. Epidemiological studies have shown a positive association between OSA and AS [6-8]. OSA was also reported to play an important role in accelerating AS plaque formation. The most prominent pathophysiological feature of OSA is intermittent hypoxia (IH), which

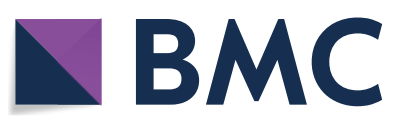

C The Author(s) 2018. This article is distributed under the terms of the Creative Commons Attribution 4.0 International License (http://creativecommons.org/licenses/by/4.0/), which permits unrestricted use, distribution, and reproduction in any medium, provided you give appropriate credit to the original author(s) and the source, provide a link to the Creative Commons license, and indicate if changes were made. The Creative Commons Public Domain Dedication waiver (http://creativecommons.org/ publicdomain/zero/1.0/) applies to the data made available in this article, unless otherwise stated. 
results from repeated episodes of upper airway obstruction. Accumulating pieces of evidence show a correlation between IH and AS [9-12]. A study has found that IH can trigger multi-pathogenic factors, especially inflammation and dyslipidemia [10]. However, whether $\mathrm{IH}$ is related to plaque development and vulnerability is still not fully clear, and the detailed mechanisms involved remain unknown.

Chronic inflammation is now regarded as a common pathway for deteriorating AS plaque [13]. Toll-like receptor 4 (TLR4), a typical representative of patternrecognition receptors in innate immune responses [14], plays an important role in activation of inflammation in AS. TLR4 signaling in AS is implicated in activation of inflammation and lipid accumulation [15], which are all associated with plaque progression and vulnerability. Furthermore, several studies indicated that TLR4 signaling may be involved in the IH-triggered inflammation $[16,17]$. Patients with OSA were also found to have higher expression of TLR4 on monocytes [18]. Nevertheless, it is still unclear whether activation of the TLR4 signaling participates in $\mathrm{IH}$-induced AS progression and vulnerability of plaque.

In this study, we investigated the role of TLR4 signaling in $\mathrm{IH}$-mediated AS progression in an apolipoprotein E-deficient $\left(\mathrm{ApoE}^{-1-}\right)$ mouse model.

\section{Methods}

\section{Experimental animals}

Sixty ApoE ${ }^{-1-}$ mice on the C57BL/6 background (Male, 8 weeks old) were purchased from Beijing Vital River
Laboratory Animal Technology Co., Ltd. (Beijing, China). Mice were fed on a high-fat diet (HFD) $(0.25 \%$ cholesterol and 15\% cocoa butter) starting at 8 weeks of age.

\section{Recombinant lentivirus and gene transfection}

Three short hairpin RNA (shRNA) sequences targeting mouse TLR4 gene (NM_021297.3) were designed and constructed. After RNAi screening, the most effective shRNA sequences (sense: 5'-GATCC GCACT CTTGA TTGCA GTTTC ATTCA AGAGA TGAAA CTGCA ATCAA GAGTG CTTTT TTG-3' anti-sense: 5'-AATTC AAAAA AGCAC TCTTG ATTGC AGTTT CATCT CTTGA ATGAA ACTGC AATCA AGAGT GCG-3'), which exhibited $89.5 \%$ of TLR4mRNA reduction, was selected for the study. Recombinant shRNATLR4 lentivirus (LV-TLR4i) was generated and purified. LV-enhanced green fluorescent protein (LV-EGFP) viral suspension was prepared, which was purchased from Geneche (Shanghai, China).

All $\mathrm{ApoE}^{-1-}$ mice were randomly divided into four groups: Sham $(n=15)$, IH $(n=15)$, IH + LV-EGFP (lentivirus-enhanced green fluorescent protein) $(\mathrm{n}=15)$ and $\mathrm{IH}+\mathrm{LV}$-TLR4i (TLR4 interference mediated by lentivirus) $(n=15)$ groups. The animal grouping and timeline of the experimental protocol are presented in Fig. 1. At 20 weeks of age, IH + LV-EGFP and IH + LV-TLR4i group mice were injected respectively with LV-EGFP and LVTLR4i $\left(2.0 \times 10^{7}\right.$ infection unit per mouse) through the tail vein, while normal saline was injected into the other two groups of mice which served as vehicle controls.

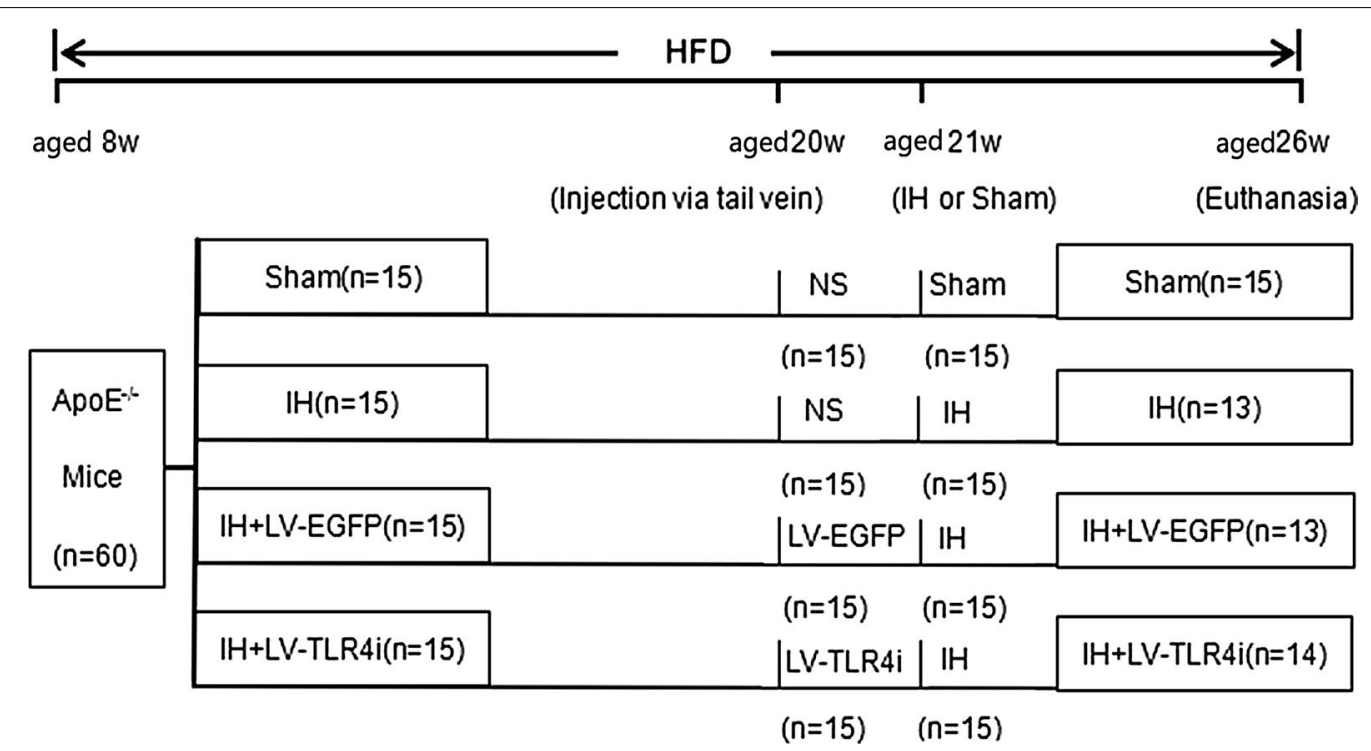

Fig. 1 Timeline of the experimental protocol in vivo. HFD high-fat diet, $w$ weeks, Sham continuous air, IH intermittent hypoxia, LV lentivirus, LV-EGFP LV-enhanced green fluorescent protein, LV-TLR4iTLR4 gene interference (TLR4i) mediated by lentivirus, NS normal saline 


\section{IH exposure}

One week after gene transfection, mice were exposed to either Sham or IH. A computer-controlled solenoid valve system was used to control the oxygen concentration by regulating the infusion of nitrogen, room air and oxygen into the exposure chamber. During each cycle of $\mathrm{IH}$, the fractional oxygen concentration of the exposure chamber was reduced to a nadir of 5.5-6.5\%, stabilized at that level for $10 \mathrm{~s}$ and increased to $20-21 \%$ in the subsequent $30 \mathrm{~s}$ $[9,10,16]$. The Sham group mice were exposed to a similar environment but only room air was used during the IH exposure time (08:00-17:00 daily) to coincide with the animals' diurnal sleep period.

Oxygen concentration was measured by oxygen Detector AR8100 (SMART SENSOR, Hong Kong).

\section{Blood pressure}

At these two time points of Pre- and Post- $\mathrm{IH}$, blood pressure was measured by the tail-cuff method (CODA2, Kent Scientific, Torrington, CT) [19].

\section{Tissue preparation and collection}

At the end of exposure protocols, mice fasted for $8 \mathrm{~h}$ before euthanasia. Arterial blood was obtained by direct cardiac puncture under $1 \%$ pentobarbital anesthesia. The heart with the aortic root, liver, kidney and the artery from the aortic arch to the left and right common iliac artery were quickly harvested and fixed in $4 \%$ paraformaldehyde ( $\mathrm{pH} 7.0-7.4)$ or frozen in liquid nitrogen. Serial cryostat Sects. ( $5 \mu \mathrm{m}$ thickness) of the aortic root were prepared by the freezing microtome (Leica CM1950, Nussloch, Germany), and were used for histological and immunohistochemical staining.

\section{Atherosclerotic lesions and immunohistochemistry analysis}

To measure overall load and distribution of AS in the inner wall of the aorta, en face lesion was stained with Oil-Red O. Moreover, the cryostat sections were stained with hematoxylin and eosin (H\&E) according to a standard protocol of our laboratory. Oil-Red $\mathrm{O}$ staining and Sirius red staining were used to detect the content of lipids and collagen of aortic root plaques, respectively. TLR4, NF-kB p65 (nuclear factor карpa B p65), $\alpha$-SMA ( $\alpha$-smooth muscle actin), MOMA-2 (macrophages/monocytes) expression in aortic root were detected by immunohistochemical staining. Briefly, cryosections were prepared, hydrated, blocked in blocking solution, and incubated with anti-TLR4 (Abcam, ab120684-1, 1:200), anti-p65 (Abcam, ab7970, 1:250), anti- $\alpha$-SMA (Abcam, ab5694; 1:200), anti-MOMA-2 (Abcam, ab33451, 1:250) as we previously described [20]. Histological and immunohistochemical staining were analyzed with Image Pro-Plus 6.0. (IPP 6.0, Media Cybernetics, Rockville, MD, USA). Atherosclerotic plaque instability index was calculated according to the standard plaque stabilization score formula: (Oil $\mathrm{Red}^{+}$area plus MOMA- $2^{+}$area $) /\left(\alpha-\mathrm{SMA}^{+}\right.$area plus collagen $\mathrm{I}^{+}$area $)$ [21].

\section{Biochemical assays}

Serum lipid profiles, including serum total cholesterol, low-density lipoprotein cholesterol (LDL-C), high-density lipoprotein cholesterol (HDL-C), total triglycerides (TG) and glucose concentrations were measured by enzymatic assay using an automatic biochemical analyzer (Roche Cobas Integra 800, Basel, Switzerland).

\section{Enzyme-linked immunosorbent assay (ELISA)}

Mice serum levels of interleukin-6 (IL-6) and tumor necrosis factor alpha (TNF- $\alpha$ ) were measured using ELISA kits (eBiosciences) according to the instructions.

\section{Cell culture and treatment protocol}

Human umbilical vein endothelial cells (HUVECs) were purchased from CHI SCIENTIFIC Biotechnology (Jiangyin, China). Cells were cultured in Dulbecco's modified Eagle medium (DMEM) supplemented with $10 \%$ fetal bovine serum (FBS; Gibco Inc.), penicillin $(100 \mathrm{U} / \mathrm{mL})$ and streptomycin $(100 \mu \mathrm{g} / \mathrm{mL})$.

Before exposure to hypoxia/reoxygenation (H/R), we first performed cell transfection. Lentivirus expressing either nonsense shRNA or anti-TLR4 shRNA were transfected into the HUVECs. HUVECs were plated at a density of $3 \times 10^{5}$ cells per $35 \mathrm{~mm}$ plate and cultured for $12 \mathrm{~h}$ before transfection. Then medium containing packaged lentivirus was added and $12 \mathrm{~h}$ later was replaced with fresh medium. After $48 \mathrm{~h}$, the interference effect of TLR4 protein was detected by using western blot for each experiment.

Hypoxia was induced in a Whitley $\mathrm{H} 35$ Hypoxystation equipment of $\mathrm{CO}_{2} / \mathrm{O}_{2}$ incubator for hypoxia research. Briefly, cells were cultured in $35 \mathrm{~mm}$ dishes at $1 \% \mathrm{O}_{2}$ for $6 \mathrm{~h}$ and then reoxygenationed at the time periods of 2, 12 and $24 \mathrm{~h}$, respectively. Control cells were cultured in normoxia in the same incubator and harvested at the specified times.

\section{Western blot analysis}

Proteins were extracted from treated HUVECs and $\mathrm{ApoE}^{-/-}$mice aortas. All samples were lysed on ice for $30 \mathrm{~min}$ in a RIPA buffer (Beyotime, Nantong, China) after being ground with a pestle. Equal amounts of protein were separated by SDS-PAGE and transferred to PVDF membranes. After blocking by $5 \%$ non-fat milk, the membranes were incubated with primary antibodies 
for TLR4 (Abcam, ab120684-1, 1:500), p65 (Abcam, ab7970, 1:1000), interleukin-1 $\beta$ (IL-1 $\beta$ )(Abcam, ab9722, 1:500) and p-p65 (Ser536) (Cell signaling, \#3033, 1:1000) overnight at $4{ }^{\circ} \mathrm{C}$. After washing and incubating with an HRP-conjugated secondary antibody $(1: 10,000)$, the immunoreactive bands were visualized using chemiluminescence (Millipore Corporation, Billerica, MA, USA). Sample loadings were normalized to $\beta$-actin expression.

\section{Quantitative real-time PCR (qRT-PCR)}

Total RNA was extracted using RNAiso Plus reagent (TaKaRa Biotech Corporation, Dalian, China) and treated with RNase-free gDNA Eraser to remove traces of genomic DNA. Complementary DNA was generated with a PrimeScript ${ }^{\mathrm{TM}}$ RT reagent kit with gDNA Eraser (TaKaRa Biotech Co.). A SYBR ${ }^{\circledR}$ Premix Ex Taq ${ }^{\text {TM }}$ Kit (TaKaRa Biotech Co.) was used for a real-time polymerase chain reaction (PCR) reaction examination. The sequences of primers $\left(5^{\prime}-3^{\prime}\right)$ were (1) for TLR4: ATGGC ATGGC TTACA CCACC (forward) and GAGGC CAATT TTGTC TCCAC A (reverse); (2) for $\beta$-actin: CACTG TGCCC ATCTA CGA (forward) and GTAGT CTGTC AGGTC CCG (reverse). The relative expression of the target gene was normalized against $\beta$-actin and the data were analyzed by the $2^{-\Delta \Delta \mathrm{CT}}$ method [22].

\section{Statistical analysis}

The data are shown as mean \pm SEM. Continuous data for two group differences were analyzed by Student's $t$ test and differences among groups were analyzed by one-way ANOVA with post hoc analysis. A p value $<0.05$ was considered statistically significant. Data were analyzed using SPSS 17.0 software (SPSS Inc., Chicago, IL, USA).

\section{Results}

IH exposure accelerated atherosclerotic plaque growth relying on the expression of TLR4 in $\mathrm{ApoE}^{-1-}$ mice

A growing number of in vivo studies show that IH exposure triggers multiple proatherogenic factors [9-12]. However, the impact of $\mathrm{IH}$ on plaque growth and its underlying mechanisms remains poorly understood. Thus, we observed the consequence of $\mathrm{IH}$ exposure in affecting atherosclerotic plaque growth by using special staining techniques. As expected, general Oil Red $\mathrm{O}$ staining showed that arteries, crossing from the aorta arch to the common iliac artery, contained extensive atherosclerotic lesions throughout the artery trunk after 5-weeks of IH exposure (Fig. 2a, b). Moreover, the average size of plaque areas in the IH group was increased 2.5- to threefold than in the Sham group (Fig. 2c, d).

To further explore whether TLR4 signaling was involved in IH-mediated atherosclerotic plaque growth, mice were transfected with TLR4 specific shRNA and then treated with $\mathrm{IH}$ exposure. Compared with the $\mathrm{IH}+\mathrm{LV}$-EGFP group, the IH +LV-TLR4i group mice

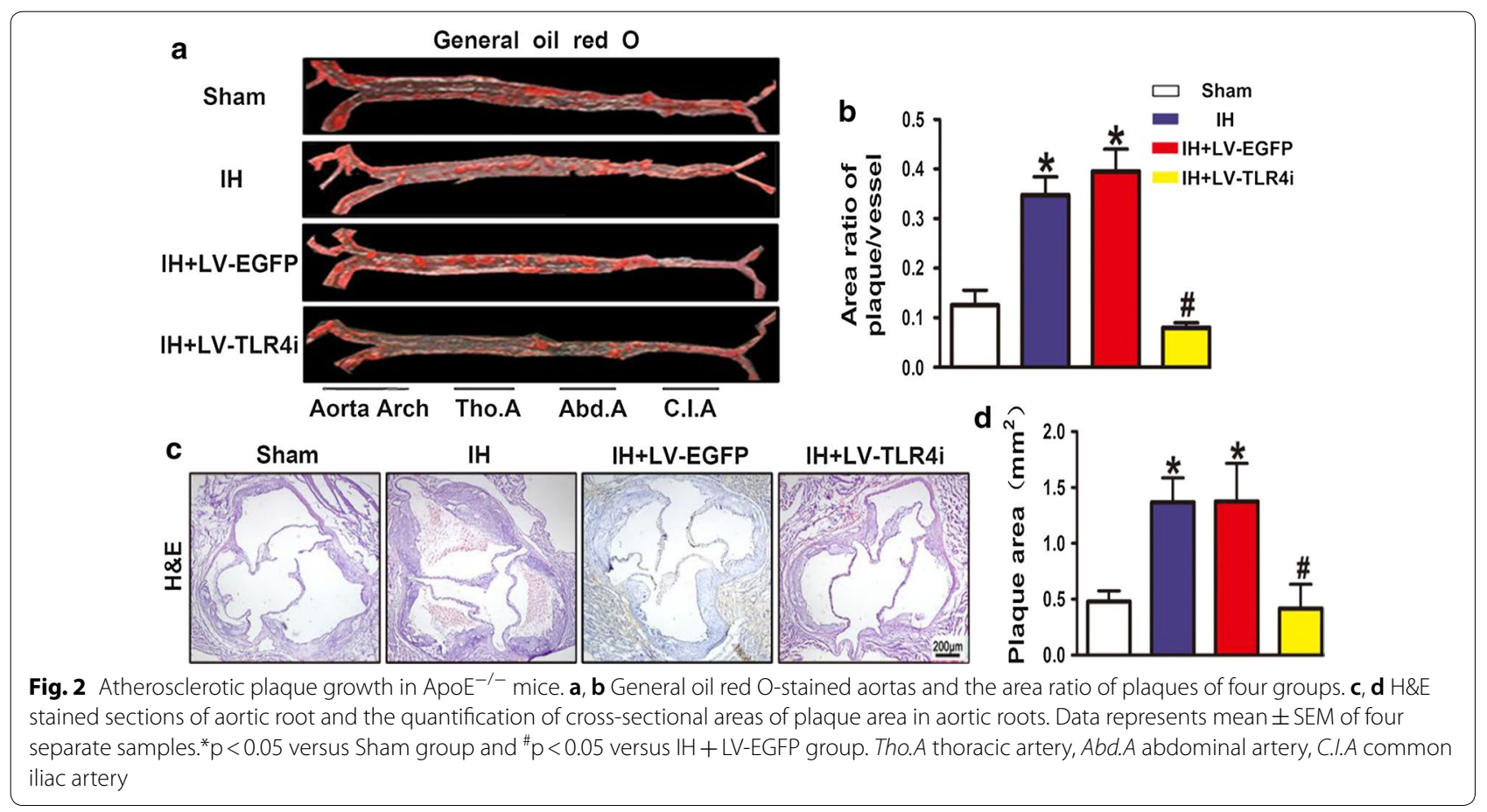


showed fewer atherosclerotic lesions (Fig. 2a, b) and smaller plaque areas (Fig. 2c, d). Therefore, these findings showed that TLR4 signaling was involved in $\mathrm{IH}$-triggered atherosclerotic plaque growth.

\section{IH-mediated TLR4 expression promoted atherosclerotic plaque instability in vivo}

Finding that $\mathrm{IH}$ accelerated atherosclerotic plaque growth in $\mathrm{ApoE}^{-1-}$ mice, we further investigated the effect of $\mathrm{IH}$ on atherosclerotic plaque vulnerability. A vulnerable plaque is characterized by a large lipid core covered with a thin fibrous cap, containing sparse smooth muscle cells and extensive macrophages [23]. In the present study, $\mathrm{IH}$ exposure increased the content of macrophages and lipids but reduced that of smooth muscle cells and collagen I in the atherosclerotic plaque, thus contributing to increased plaque vulnerability in ApoE $^{-1-}$ mice (Fig. 3a-d). The plaque vulnerability index in the IH group was increased in several times relative to the Sham group (Fig. 3e). However, mice transfected with TLR4-specific shRNA showed a more stable atherosclerotic plaque (Fig. 3a-e). These changes in the composition of plaques indicated that $\mathrm{IH}$ exposure promotes plaque instability and the potential for plaque rupture. In addition, TLR4 interference modified the composition of plaques and improved the stability of vulnerable plaque in $\mathrm{IH}$-exposed $\mathrm{ApoE}^{-/-}$mice.

\section{IH exposure exacerbated inflammatory response via TLR4-mediated signaling in $\mathrm{ApoE}^{-1-}$ mice}

TLR4 signaling is closely involved in the activation of the inflammatory response. To investigate whether TLR4 signaling involved in IH-mediated plaque development by aggravating inflammatory activity in vivo, we first examined TLR4 expression in the aortic plaque in $\mathrm{IH}$-exposed $\mathrm{ApoE}^{-1-}$ mice. IH upregulated both protein (Fig. 4a, c) and mRNA (Fig. 4f) levels of TLR4 in the aorta. Meanwhile, the nuclear localization of p65 (Fig. 4b) and the phosphorylation of p65 (Fig. 4d) in the IH group were significantly increased than that in the Sham group. Moreover, IH upregulated the protein level of IL-1 $\beta$ (Fig. 4e) and the infiltration of macrophages (Fig. 3b) in atherosclerotic plaque, as well as increased serum levels of IL- 6 and TNF- $\alpha$ in ApoE ${ }^{-1-}$ mice (Fig. 4g, h).

To explore possible mechanisms by which IH promoted the inflammatory response, we further elaborated the relationship between TLR4 signaling and inflammatory activity in $\mathrm{IH}$-exposed $\mathrm{ApoE}^{-1-}$ mice. Compared with the IH + LV-EGFP group, TLR4 interference evidently reduced TLR4 expression (Fig. 4a, c, f) and activation of p65 (Fig. 4b, d) in the IH + LV-TLR4i group mice aortas. Moreover, inhibition of TLR4 could restore IL-1 $\beta$,
IL-6, and TNF- $\alpha$ to the levels of the Sham group (Fig. 4e, $\mathrm{g}, \mathrm{h})$. Likewise, TLR4 interference effectively blocked the increasing of macrophage infiltration in $\mathrm{IH}$-exposed atherosclerotic plaque (Fig. 3b). Consequently, we concluded that IH accelerated plaque progression via TLR4-triggered vascular and systemic inflammatory responses.

\section{TLR4 interference restored IH-induced body weight loss but had no effect on blood pressure elevation in $\mathrm{ApoE}^{-/-}$ mice}

The results of body weight and blood pressure of the four groups are presented in Table 1. There are no differences in body weight among the four groups at baseline $\left(\mathrm{V}_{0}\right)$. After 5 weeks of IH exposure, IH and IH+LV-EGFP groups were significantly reduced in body weight $\left(\mathrm{V}_{1}\right)$ compared with the Sham group (Table), but this effect of IH on body weight was reversed by TLR4 interference, as shown in Table 1.

At baseline, there were no differences in systolic blood pressure (SBP) and diastolic blood pressure (DBP) among the four groups. After IH exposure, SBP and DBP were significantly increased compared with the Sham group. However, there were no significant differences between the IH + LV-TLR4i group and the IH + LV-EGFP group (Table 1), indicating that TLR4 interference had little effect on IH-elevated blood pressure.

\section{The role of TLR4 in IH-induced glycolipid disorders in vivo} As most of the OSA patients are complicated with dyslipidemia, we investigated whether IH had an effect on glycolipid metabolisms. As shown in Fig. 5a-e, IH significantly increased serum levels of total cholesterol, LDL-C, HDL-C, glucose, with no influence on TG levels. TLR4 interference reversed the $\mathrm{IH}$-induced increase of total cholesterol and LDL-C, but had little influence on the serum levels of HDL-C, TG and glucose. Therefore, IH could cause glycolipid metabolism disorders and TLR4 was involved in $\mathrm{IH}$-mediated hypercholesterolemia.

\section{Essential role of TLR4 in H/R activated proinflammatory signaling in vitro}

To further prove that $H / R$ exposure could trigger the activation of the proinflammatory TLR4/NF- $\mathrm{kB}$ signaling, we applied HUVECs as the carrier of in vitro study. Compared with the other $H / R$ intervals, we discovered that the protein level of TLR4 was significantly higher in the H6/R12 (6 h of hypoxia and $12 \mathrm{~h}$ of reoxygenation) group (Fig. 6a). TLR4 expression both at the gene level (Fig. 6b) and protein level (Fig. 6c) was significantly higher in H6/R12 group than in the Control group. Additionally, H6/R12 exposure also promoted the phosphorylation of p65 (Fig. 6d). To further 


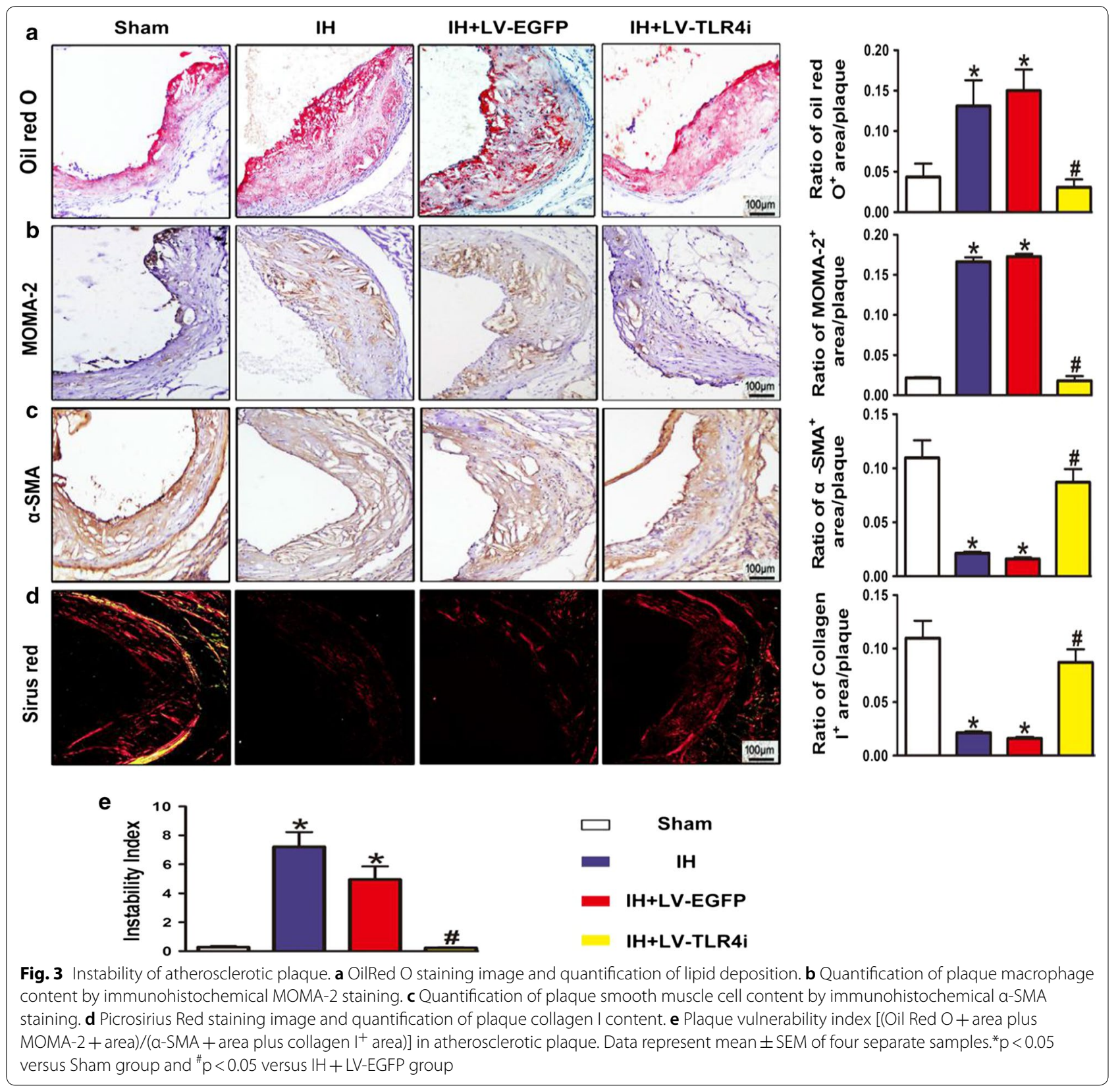

investigate the role of TLR4 in H/R-triggered p65 activation, we added treatment with TLR4-shRNA. We found that TLR4 interference reversed the increase of TLR4 expression (Fig. 6b, c) and the phosphorylation of p65 (Fig. 6d). These data indicated that H/R exposure significantly enhanced the activation of the proinflammatory TLR4/NF- $\mathrm{kB}$ signaling in HUVECs, and TLR4 interference mitigated inflammatory-related endothelial dysfunction.

\section{Discussion}

In this study, we found that $\mathrm{IH}$ increased the instability of atherosclerotic plaques. After TLR4 interference, the instability of plaques was decreased with attenuated inflammation and dyslipidemia.

AS is a multifactorial systemic disease of large and medium arteries. It is gradually recognized that plaques with a rapid progression rate have a much greater probability of resulting in ACS [24]. Thin plaque fibrous caps, severe inflammatory infiltration and high lipid content 


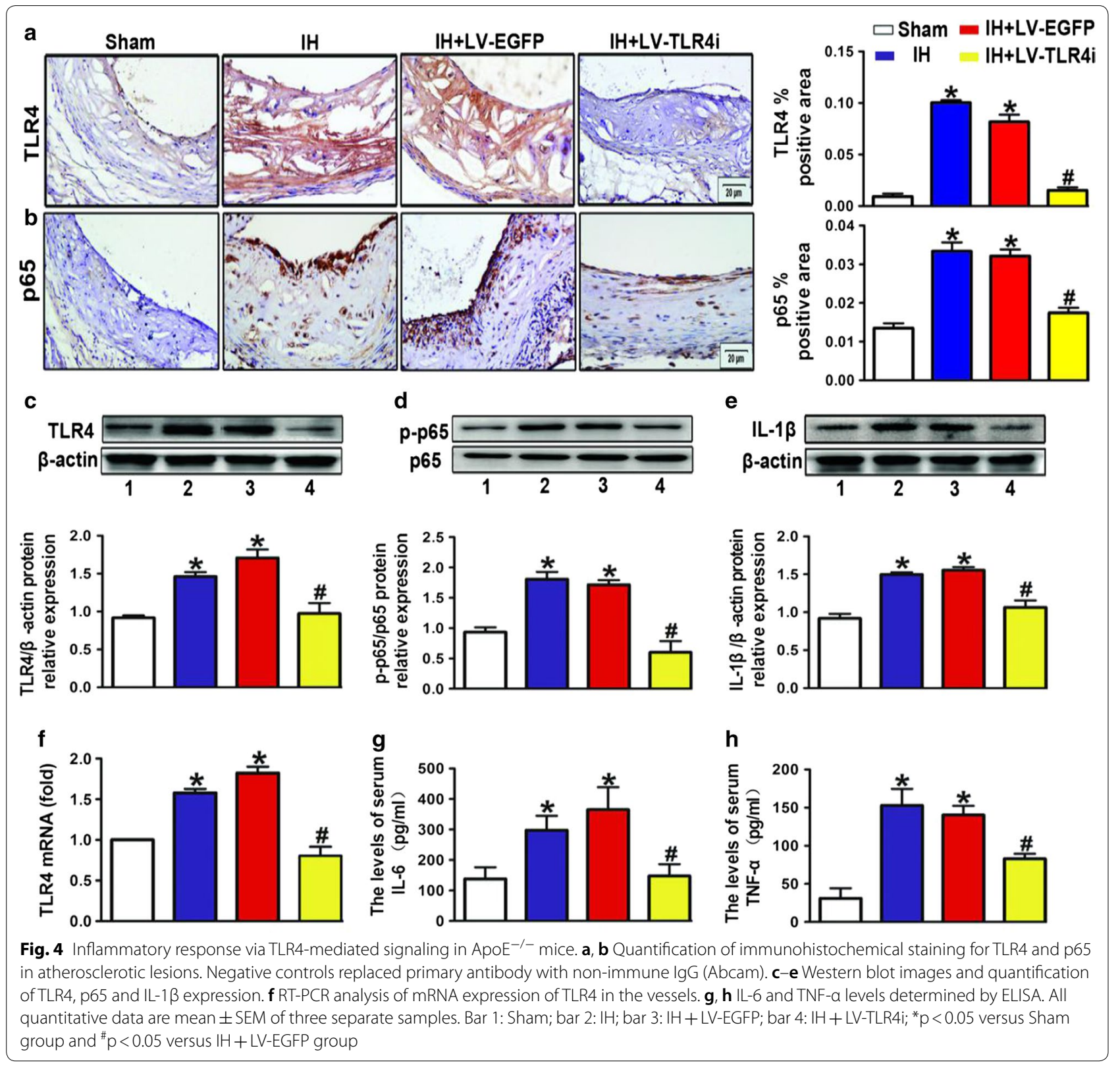

Table 1 Body weight, weight loss and systemic blood pressure in $\mathrm{ApoE}^{-1-}$ mice

\begin{tabular}{|c|c|c|c|c|c|c|c|}
\hline \multirow[t]{2}{*}{ Groups } & \multicolumn{3}{|c|}{ Body weight (g) } & \multicolumn{4}{|c|}{ Blood pressure $(\mathrm{mmHg})$} \\
\hline & Pre-IH & After-IH & WL & Pre-IH & Pre-IH & After-IH & After-IH \\
\hline- & (V0) & (V1) & $(\mathrm{V} 0-\mathrm{V} 1)$ & (SBP) & (DBP) & (SBP) & (DBP) \\
\hline Sham & $30.77 \pm 0.47$ & $28.94 \pm 1.06$ & $1.84 \pm 0.81$ & $116.67 \pm 2.56$ & $73.90 \pm 3.81$ & $102.89 \pm 0.90$ & $59.67 \pm 1.96$ \\
\hline $\mathbb{H}$ & $30.64 \pm 0.31$ & $24.57 \pm 0.74^{*}$ & $6.06 \pm 0.69^{*}$ & $116.47 \pm 1.79$ & $73.24 \pm 1.19$ & $129.89 \pm 2.11^{*}$ & $81.07 \pm 4.05^{*}$ \\
\hline $\mathrm{IH}+\mathrm{LV}$-EGFP & $30.79 \pm 0.28$ & $24.39 \pm 0.93^{*}$ & $6.40 \pm 1.14^{*}$ & $121.90 \pm 2.52$ & $71.62 \pm 2.27$ & $123.11 \pm 2.42^{*}$ & $76.77 \pm 2.99^{*}$ \\
\hline$I H+L V-T L R 4 i$ & $31.57 \pm 0.42$ & $30.36 \pm 0.55^{\#}$ & $1.21 \pm 0.38^{\#}$ & $123.43 \pm 2.89$ & $77.47 \pm 2.35$ & $127.00 \pm 2.87^{*}$ & $80.00 \pm 2.94^{*}$ \\
\hline
\end{tabular}

Results are expressed as mean \pm SEM and $n=6$ per group

$W L$ weight loss, $V 0$ pre-IH body weight, $V 1$ after-IH body weight

${ }^{*} p<0.05$ versus Sham group and ${ }^{\#} p<0.05$ versus IH + LV-EGFP group 

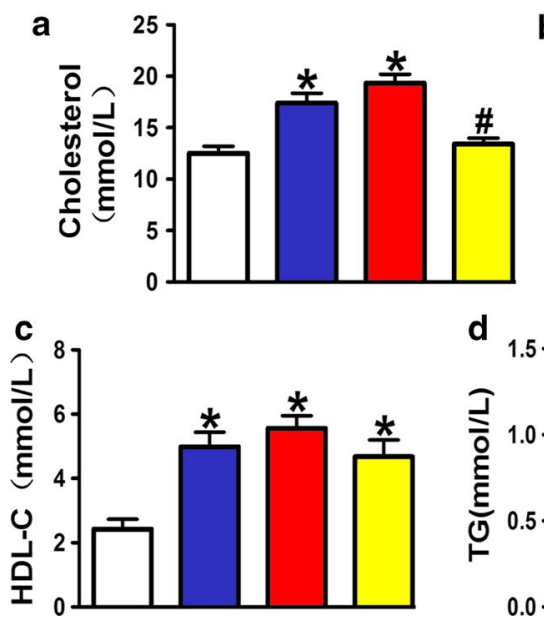


e



Fig. 5 Glycolipid disorder in vivo. a-e Total cholesterol $(n=6)$, high-density lipoprotein cholesterol $(H D L-C)(n=6)$, low-density lipoprotein cholesterol $(\operatorname{LDL}-C)(n=6)$, triglycerides $(T G)(n=6)$ and blood glucose $(n=6)$ in $A_{00 E^{-1-}}$ mice. ${ }^{*} p<0.05$ versus Sham group and ${ }^{\#} p<0.05$ versus




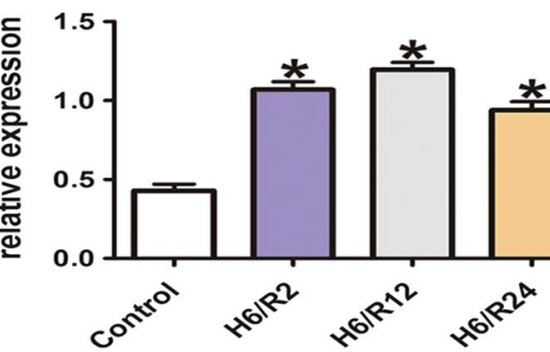

C

\section{TLR4}

$\beta$-actin
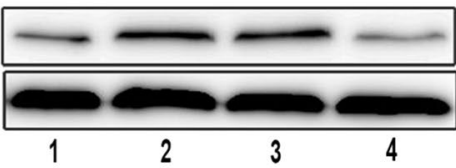

등
$\mathrm{IH}+$ LV-EGFP group

a

\section{(1)}
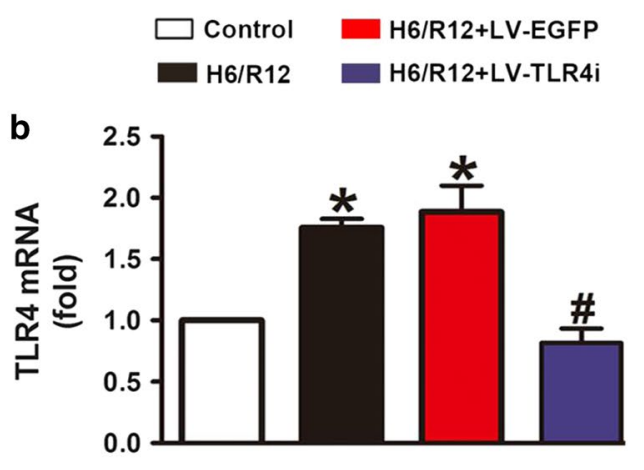

d
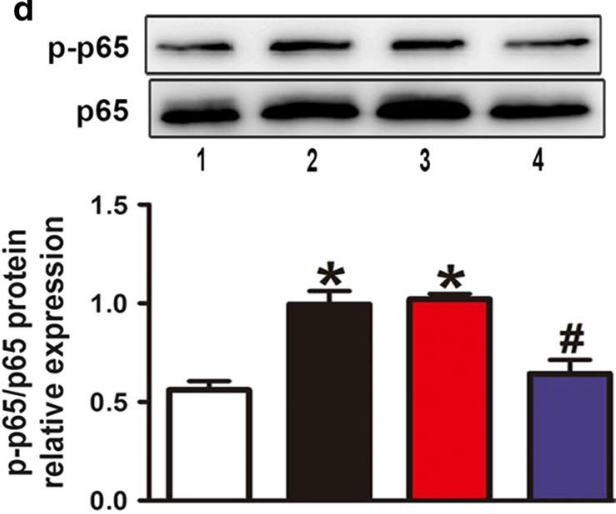

Fig. 6 Essential role of TLR4 in H/R exposure activated proinflammatory signaling in vitro. a Western blot images and quantification of TLR4 in HUVECs with distinct time periods of reoxygenation exposure. $\mathbf{b}$ Effects of $\mathrm{H} / \mathrm{R}$ exposure induced mRNA expression of TLR4 and TLR4 interference reversed these effects in HUVECs. $\mathbf{c}$, d Western blot images and quantification of TLR4 and p-p65 expression in treated HUVECs. All quantitative data are mean \pm SEM of three separate samples. Bar 1: Control; bar 2: H6/R12; bar 3: H6/R12 + LV-EGFP; bar 4: H6/R12 + LV-TLR4i; *p $<0.05$ versus Control group and $\# p<0.05$ versus H6/R12 + LV-EGFP group. H6/R2, Reoxygenation $2 \mathrm{~h}$ after $6 \mathrm{~h}$ of hypoxia; H6/R12, Reoxygenation $12 \mathrm{~h}$ after $6 \mathrm{~h}$ of hypoxia; H6/R24, Reoxygenation $24 \mathrm{~h}$ after $6 \mathrm{~h}$ of hypoxia 
are known to make plaque vulnerable [23]. OSA has been newly recognized as an underlying risk factor of AS [2, 3]. IH is the most prominent pathophysiological feature of OSA [25]. In the present study, we demonstrated that $\mathrm{IH}$ increased atherosclerotic plaque growth and vulnerability in $\mathrm{ApoE}^{-/-}$mice, with extensive macrophage infiltration and increased lipid content, and reduced content of smooth muscle cells and collagen.

Progressive vascular and systemic chronic inflammation are the common pathways to AS plaque deterioration [26]. OSA has been proved to be related to chronic systemic inflammation and metabolic syndrome [27]. A meta-analysis has confirmed that the levels of systemic inflammatory markers, such as IL- 6 and TNF- $\alpha$, were higher in OSA patients compared with controls [28]. Likewise, data obtained from animal experiments also supported these results $[10,11]$. In our study, IH elevated serum concentrations of IL- 6 and TNF- $\alpha$, and the content of IL-1 $\beta$ and macrophage infiltration in AS plaques in $\mathrm{ApoE}^{-1-}$ mice. Inflammation in atherosclerotic plaque attenuated the tensile strength of the collagen cap, enhanced cell death and reinforced prothrombotic activity, and eventually caused ACS [26]. Moreover, inflammation recruited macrophages and resulted in foam cells derived from macrophages accumulating in plaque.

NF- $\mathrm{kB}$ is a key transcription factor of inflammation. TLR4, a typical pattern-recognition receptor, is the upstream gene of NF-kB and has been confirmed to be closely related to inflammatory activation in plaques [15]. Accumulating pieces of evidence also showed that TLR4 is deeply implicated in activation of inflammation in patients with OSA [18].

As we've expected, along with increased TLR4 expression, enhanced nuclear localization of p65 and the phosphorylation of $\mathrm{p} 65$, and the inflammation factors were observed in the IH treated group. After TLR4 interference, vascular and systemic inflammation was greatly attenuated. Furthermore, H/R exposure promoted TLR4 expression and activation of TLR4/NF- $\mathrm{KB}$ signaling in endothelial cells, while TLR4 interference reversed these effects. As a result, the atherosclerotic loads and atherosclerotic plaque vulnerability were decreased more than $50 \%$ after TLR4 interference. Accordingly, we conclude that $\mathrm{IH}$ promotes the plaque growth and instability by triggering the activation of TLR4/NF- $\mathrm{kB}$ signaling and the downstream proinflammatory cascade responses. Therefore, TLR4 maybe an ideal target for interfering in the OSA-induced AS progression.

We also found $\mathrm{IH}$ exposure increased serum levels of total cholesterol and LDL-C. After TLR4 interference, IH-induced total cholesterol and LDL-C elevation had been significantly reversed. Previous studies reported that $\mathrm{IH}$ may cause atherogenesis, to a large extent, depending on dyslipidemia [10]. The IH induced dyslipidemia may play an important role in $\mathrm{IH}$-mediated AS plaque progression in the present experiment. It was also reported that $\mathrm{IH}$ exposure down-regulates scavenger receptor class B1 (SR-B1) protein expression, disturbs cholesterol clearance and enhances cholesterol accumulation by activating NF- $\mathrm{kB}$ [29]. Activating NF- $k B$ down-regulates SR-B1 and ATP-binding cassette transporter $\mathrm{A} 1$ (ABCA1) expression [9]. It is inferred that TLR4 interference may decrease the total cholesterol and LDL-C by inhibiting the activity of NF-кB. TLR4 signaling may also play an important role in $\mathrm{IH}$-mediated AS plaque progression by aggravating dyslipidemia.

In the present study, we used $\mathrm{ApoE}^{-1-}$ mice as an animal model because it is more apt to show the pathological changes of OSA-accompanying AS, such as inflammatory susceptibility, dyslipidemia and extracellular matrix degradation [30-32]. To highlight the effect of IH but not a high cholesterol diet on plaque progression, we fed $\mathrm{ApoE}^{-/-}$mice with a special highfat diet (HFD), in which the content of cholesterol $(0.25 \%)$ was one-fifth of the previous study $(1.25 \%)$ at the beginning of our study $[9,12]$. Then, mice were exposed to $\mathrm{IH}$ at the age of the formation of spontaneous early stage atherosclerotic lesions. This model may provide a more intuitive understanding of the process of OSA-accompanying AS development.

\section{Conclusions}

With this modified animal model, we found that IH accelerated growth and vulnerability of atherosclerotic plaque, which probably acted by triggering the activation of TLR4/NF- $\mathrm{kB}$ signaling. We further prove the role of TLR4 in the H/R-triggered activation of the proinflammatory TLR4/NF-kB signaling in endothelial cells. To the 


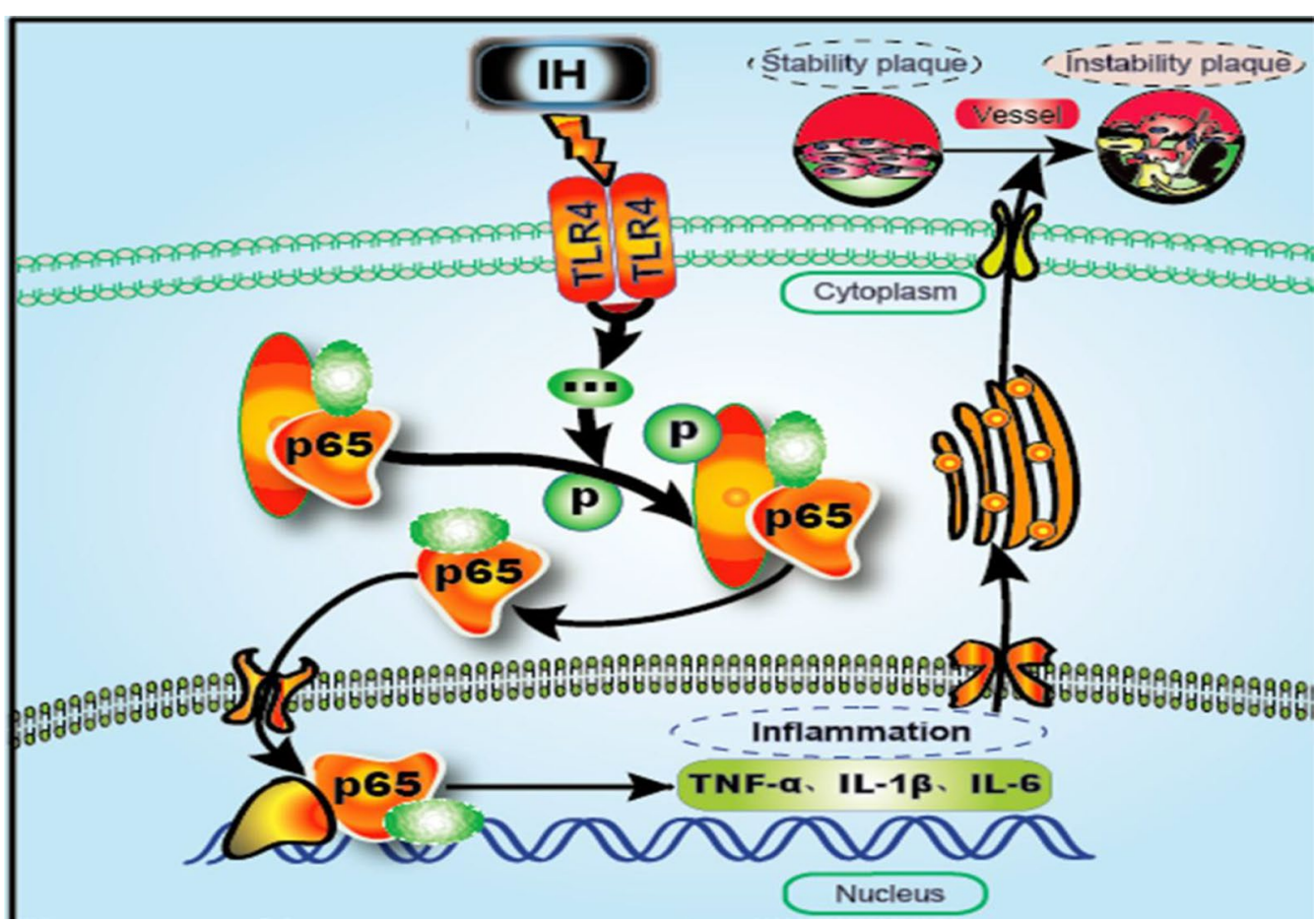

Fig. 7 Possible mechanism diagram of $\mathrm{IH}$ accelerated atherosclerotic plaque growth and instability. IH exposure enhanced the expression of TLR4 and facilitated the activation of NF-KB p65 both in vivo and in vitro. Activated NF-KB entered the nucleus and augmented the expression of inflammatory cytokines. Inflammatory activity accelerated atherosclerotic plaque growth and instability. In summary, IH promotes atherosclerosis progression mainly through activating inflammatory response, which depends on TLR4/NF-KB signaling

best of our knowledge, it is the first report that presents substantial evidence about IH-induced AS growth and vulnerability and the detailed mechanism, in which TLR4 is involved (Fig. 7). These findings may provide a new insight into the treatment of $\mathrm{IH}$-induced AS progression.

\section{Abbreviations}

AS: atherosclerosis; OSA: obstructive sleep apnea; ACS: acute coronary syndrome; IH: intermittent hypoxia; TLR4: toll-like receptor 4; NF-kB: nuclear factor kappa B; $\mathrm{ApoE}^{-1-}$ : apolipoprotein E-deficient; HFD: high-fat diet; EGFP: enhanced green fluorescent protein; SBP: systolic blood pressure; DBP: diastolic blood pressure; a-SMA: a-smooth muscle actin; IL-6: interleukin-6; TNF-a: tumor necrosis factor alpha; MOMA-2: macrophages/monocytes; shRNA: short hairpin RNA; H/R: hypoxia/reoxygenation.

\section{Authors' contributions}

Conceived and designed the experiments: LHL, LHX, ZXQ and JZ. Performed the experiments: ZXQ, GR and DM. Analyzed the data: LHL, LHX, ZXQ. Contributed reagents/materials/analysis tools: ZXQ, GR, JZ and DM. Wrote the paper: LHL, LHX, ZXQ and JZ. All authors read and approved the final manuscript.

\section{Author details}

${ }_{1}^{1}$ Department of Cardiology, The Second Affiliated Hospital of Fujian Medical University, Quanzhou 362000, Fujian, People's Republic of China. ${ }^{2}$ The Key Laboratory of Cardiovascular Remodeling and Function Research, Chinese Ministry of Education and Chinese Ministry of Health, The State and Shandong Province Joint Key Laboratory of Translational Cardiovascular Medicine, Department of Cardiology, Qilu Hospital of Shandong University, No. 107, Wen Hua Xi Road, Jinan 250012, Shandong, China. ${ }^{3}$ Rutgers Robert Wood Johnson Medical School, New Jersey, New Brunswick, USA. ${ }^{4}$ Department of Cardiology, Ji'an Municipal Center People's Hospital, Ji'an, Jiangxi, China.

\section{Acknowledgements}

We are thankful to Shanying Huang, Zhonghua Qu for suggestions and technical contributions and Dr. Julia Zheng for English editing.

\section{Competing of interests}

The authors declare that they have no competing interests.

\section{Availability of data and materials}

All data generated or analysed during this study are included in this publication.

\section{Consent for publication}

Not applicable.

\section{Ethics approval and consent to participate}

All animal care procedures and experimental protocols were approved by the Animal Care Committee of Qilu Hospital of Shandong University and complied with the Guide for the Use and Care of Laboratory Animals published by the US National Institutes of Health (NIH publication 80-23, revised 1996).

\section{Funding}

This work was supported by the National Natural Science Foundation of China (No. 81100152), the Key Research and Development Program of Shandong Province (2017GSF218045), Fujian Provincial Natural Science Foundation (No. 2016J01441), Fujian ZQN backbone science foundation (No. 2016-ZQN-55) and Fujian Provincial Guide Project Foundation (No. 2016Y0032), Qingdao people's livelihood science and technology plan project (17-3-3-30-nsh).

\section{Publisher's Note}

Springer Nature remains neutral with regard to jurisdictional claims in published maps and institutional affiliations. 
Received: 16 October 2017 Accepted: 10 April 2018

Published online: 19 April 2018

\section{References}

1. World Health Organization, World Heart Federation, World Stroke Organization. Global atlas on cardiovascular disease prevention and control, Policies, strategies and interventions. Geneva: World Health Organization; 2011.

2. Toraldo DM, DE Nuccio F, DE Benedetto M, Scoditti E. Obstructive sleep apnoea syndrome: a new paradigm by chronic nocturnal intermittent hypoxia and sleep disruption. Acta Otorhinolaryngol Ital. 2015;35:69-74.

3. McNicholas WT, Bonsigore MR. Sleep apnoea as an independent risk factor for cardiovascular disease: current evidence, basic mechanisms and research priorities. Eur Respir J. 2007;29:156-78.

4. Flavia ACGL, Maia C. Impact of high risk for obstructive sleep apnea on survival after acute coronary syndrome: insights from the ERICO registry. Arq Bras Cardiol. 2017;108:31-7.

5. Tishler PV, Larkin EK, Schluchter MD, Redline S. Incidence of sleep-disordered breathing in an urban adult population: the relative importance of risk factors in the development of sleep-disordered breathing. JAMA. 2003;289:2230-7.

6. Bouloukaki I, Mermigkis C, Kallergis EM, Moniaki V, Mauroudi E, Schiza SE. Obstructive sleep apnea syndrome and cardiovascular disease: the influence of C-reactive protein. World J Exp Med. 2015;5:77-83.

7. Ciccone MM, Scicchitano P. The intimate association of OSAS and early systemic atherosclerosis. Respir Med. 2012;106:1623.

8. Benbir G, Karadeniz D. A pilot study of the effects of non-invasive mechanical ventilation on the prognosis of ischemic cerebrovascular events in patients with obstructive sleep apnea syndrome. Neurol Sci. 2012;33:811-8.

9. Song D, Fang G, Mao SZ, Ye X, Liu G, Gong Y, Liu SF. Chronic intermittent hypoxia induces atherosclerosis by NF-kB-dependent mechanisms. Biochim Biophys Acta. 1822;2012:1650-9.

10. Fang G, Song D, Ye X, Mao SZ, Liu G, Liu SF. Chronic intermittent hypoxia exposure induces atherosclerosis in ApoE knockout mice: role of NF-kB p50. Am J Pathol. 2012;181:1530-9.

11. Arnaud C, Poulain L, Levy P, Dematteis M. Inflammation contributes to the atherogenic role of intermittent hypoxia in apolipoprotein-E knock out mice. Atherosclerosis. 2011;219:425-31.

12. Savransky V, Nanayakkara A, Li J, Bevans S, Smith PL, Rodriguez A, Polotsky VY. Chronic intermittent hypoxia induces atherosclerosis. Am J Respir Crit Care Med. 2007;175:1290-7.

13. Libby P, Tabas I, Fredman G, Fisher EA. Inflammation and its resolution as determinants of acute coronary syndromes. Circ Res. 2014:114:1867-79.

14. Medzhitov R. Toll-like receptors and innate immunity. Nat Rev Immunol. 2001;1:135-45.

15. Yvan-Charvet LWCP. Increased inflammatory gene expression in ABC transporter deficient macrophages: free cholesterol accumulation, increased signaling via toll-like receptors and neutrophil infiltration of atherosclerotic lesions. Circulation. 2008;118:1837-47.

16. Poulain L, Richard V, Levy P, Dematteis M, Arnaud C. Toll-like receptor-4 mediated inflammation is involved in the cardiometabolic alterations induced by intermittent hypoxia. Mediat Inflamm. 2015;2015:620258.
17. Smith SM, Friedle SA, Watters JJ. Chronic intermittent hypoxia exerts CNS region-specific effects on rat microglial inflammatory and TLR4 gene expression. PLoS ONE. 2013;8:e81584.

18. Akinnusi PJTKM. Toll-like receptor activity in patients with obstructive sleep apnea. Sleep Breath. 2013;17:1009-16.

19. Li CB, Li XX, Chen YG, Zhang C, Zhang MX, Zhao XQ, Hao MX, Hou XY, Gong ML, Zhao YX, Bu PL, Zhang Y. Effects and mechanisms of PPARa activator fenofibrate on myocardial remodelling in hypertension. J Cell Mol Med. 2009:13:4444-52.

20. Ni JQ, Ouyang Q, Lin L, Huang Z, Lu H, Chen X, Lin H, Wang Z, Xu D, Zhang Y. Role of toll-like receptor 4 on lupus lung injury and atherosclerosis in LPS-challenge ApoE(-)/(-) mice. Clin Dev Immunol. 2013;2013:476856.

21. Ni W, Egashira K, Kitamoto S, Kataoka C, Koyanagi M, Inoue S, Imaizumi K, Akiyama C, Nishida KI, Takeshita A. New anti-monocyte chemoattractant protein-1 gene therapy attenuates atherosclerosis in apolipoprotein E-knockout mice. Circulation. 2001;103:2096-101.

22. Gargiulo SEA. Relation between TLR4/NF-KB signaling pathway activation by 27-hydroxycholesterol and 4-hydroxynonenal, and atherosclerotic plaque instability. Aging Cell. 2015;14:569-81.

23. Virmani R, Burke AP, Farb A, Kolodgie FD. Pathology of the vulnerable plaque. J Am Coll Cardiol. 2006;47:C13-8.

24. Ahmadi A, Leipsic J, Blankstein R, Taylor C, Hecht H, Stone GW, Narula J. Do plaques rapidly progress prior to myocardial infarction? The interplay between plaque vulnerability and progression. Circ Res. 2015;117(1):99-104.

25. Song D, Fang G, Greenberg H, Liu SF. Chronic intermittent hypoxia exposure-induced atherosclerosis: a brief review. Immunol Res. 2015;63:121-30.

26. Hansson GK, Libby P, Tabas I. Inflammation and plaque vulnerability. J Intern Med. 2015:278:483-93.

27. Parati G, Lombardi C, Narkiewicz K. Sleep apnea: epidemiology, pathophysiology, and relation to cardiovascular risk. Am J Physiol Regul Integr Comp Physiol. 2007;293:R1671-83.

28. Nadeem R, Molnar J, Madbouly EM, Nida M, Aggarwal S, Sajid H, Naseem J, Loomba R. Serum inflammatory markers in obstructive sleep apnea: a meta-analysis. J Clin Sleep Med. 2013;9:1003-12.

29. Li J, et al. Intermittent hypoxia induces hyperlipidemia in lean mice. Circ Res. 2005;97(7):698-706.

30. Somers VK, White DP, Amin R, Abraham WT, Costa F, Culebras A, Daniels S, Floras JS, Hunt CE, Olson LJ, Pickering TG, Russell R, Woo M, Young T. Sleep apnea and cardiovascular disease: an American Heart Association/ american College Of Cardiology Foundation Scientific Statement from the American Heart Association Council for High Blood Pressure Research Professional Education Committee, Council on Clinical Cardiology, Stroke Council, and Council On Cardiovascular Nursing. In collaboration with the National Heart, Lung, and Blood Institute National Center on Sleep Disorders Research (National Institutes of Health). Circulation. 2008;118:1080-111.

31. Shaw PX. Rethinking oxidized low-density lipoprotein, its role in atherogenesis and the immune responses associated with it. Arch Immunol Ther Exp (Warsz). 2004;52:225-39.

32. Zhang SH, Reddick RL, Piedrahita JA, Maeda N. Spontaneous hypercholesterolemia and arterial lesions in mice lacking apolipoprotein E. Science. 1992;258:468-71. 